\title{
Must-Have Knowledge about the Helicobacter pylori-Negative Gastric Cancer
}

\author{
Jeong Ho Kim* and Dae Young Cheung ${ }^{\dagger}$ \\ "Department of Internal Medicine, Myongji Hospital, Seonam University College of Medicine, Goyang, and ${ }^{\dagger}$ Department of Internal Medicine, \\ The Catholic University of Korea College of Medicine, Seoul, Korea
}

See "Comparison between Resectable Helicobacter pylori-Negative and -Positive Gastric Cancers" by Hee Jin Kim, et al. on page 212-219, Vol. 10. No. 2, 2016

Are gastric cancers all attributable to Helicobacter pylori infection? This question is very fundamental for gastric cancer prevention strategies. Cancers are a result of genetic instability and the carcinogenic causes are comprised of both host and environmental factors. Infection is a major initiative for cancer development and, in 2008 statistics, the population attributable fraction (PAF) for infectious agents was 16.1\%, meaning that around 2 million new cancer cases were attributable to infections. ${ }^{1} H$. pylori, hepatitis B and C viruses, human papilloma viruses are the most well known infectious agents for human cancer. H. pylori was discovered in 1983 and approved as class I gastric carcinogen in 1994 based on the strong epidemiological evidences. Gastric cancer is highly prevalent in Korea and the national effort to reduce the socioeconomic burden from gastric cancer is a kind of desperate. National cancer screening program has been working since 1999 and which provides fully-government-paid esophagogastroduodenoscopy (EGD) examination to all Korean citizens over 40 years old. In near Korea, Japanese government began population-wide eradication of $H$. pylori in order to eradicate gastric cancer since 2013.

Most of gastric cancers present $H$. pylori infection at the time of diagnosis but, in not infrequent cases, $H$. pylori is not detected despite of thorough histological examination over the mucosa. The atrophy and metaplastic changes preceding the cancer development are environmentally harsh for $H$. pylori survival and bacterial colonization might be expelled spontaneously. However, $H$. pylori left their footprints serologically or histologically and we can recognize past $H$. pylori infection. $H$. pylori-negative gastric cancer means a gastric cancer witch developed without a contribution of $H$. pylori infection. Auto- immune gastritis, Epstein Barr virus (EBV) infection and genetic predisposition are proposed to be related to the development of $H$. pylori-negative gastric cancer. The frequency of $H$. pylorinegative gastric cancer can answer the expected impact of $H$. pylori eradication for gastric cancer eradication. And also, the characterization of $H$. pylori-negative gastric cancer may give us a new insight for gastric cancer carcinogenesis and a need for new strategies for gastric cancer prevention.

Kim and colleagues ${ }^{2}$ analyzed $H$. pylori-negative gastric cancer in Korea in the article "Comparison between resectable Helicobacter pylori-negative and -positive gastric cancer." The frequency of $H$. pylori-negative gastric cancer was $4.0 \%$ (28/705). H. pylori-negative gastric cancers showed a trend of proximal location, higher frequency of microsatellite instability high (MSI-H) and p53 overexpression, but the differences from $H$. pylori-positive gastric cancer did not reach to the statistical significance. This study seems a sequel of authors' earlier publication in $2011,{ }^{3}$ in which $H$. pylori-negative gastric cancer was $5.3 \%$ in frequency.

Studies about $H$. pylori-negative gastric cancer are reported from various regions in the world, but mostly from Korea and Japan. Japanese studies report the frequency of $H$. pylorinegative gastric cancer around 1.0\%, which is lower than that of others. ${ }^{4}$ Regarding the frequency of $H$. pylori-negative gastric cancer, the most debated part is about the definition of $H$. pylori-negative gastric cancer. Depending on the definition, the frequency of $H$. pylori-negative gastric cancer can vary in a wide range. In this study $(n=705), 68$ patients with atrophic gastritis or intestinal metaplasia were initially classified as $H$. pylorinegative gastric cancer, but authors determined to recategorize

Correspondence to: Dae Young Cheung

Department of Internal Medicine, Yeouido St. Mary’s Hospital, The Catholic University of Korea College of Medicine, 222 Banpo-daero, Seocho-gu, Seoul 06591, Korea

Tel: +82-2-3779-1519, Fax: +82-2-3779-1331, E-mail: adagio@catholic.ac.kr pISSN 1976-2283 eISSN 2005-1212 http://dx.doi.org/10.5009/gnl16002

@) This is an Open Access article distributed under the terms of the Creative Commons Attribution Non-Commercial License (http://creativecommons.org/licenses/by-nc/4.0) which permits unrestricted non-commercial use, distribution, and reproduction in any medium, provided the original work is properly cited. 
them into $H$. pylori-positive gastric cancer. The original categorization might result in the frequency of $H$. pylori-negative gastric cancer as 13.9\%. Japanese studies employed more aggressive inclusion criteria for $H$. pylori positivity and $H$. pylorinegative gastric cancer is estimated $0.42 \%{ }^{5}$ and $0.66 \%{ }^{4}$

What is the definition of $H$. pylori-negative gastric cancer? $H$. pylori infection status can be classified as current, past and possibly past infection (Table 1). Current infection means the presence of $H$. pylori in situ. Viable $H$. pylori can be detected with urea breath test, stool antigen, and four kinds of biopsy based tests including rapid urease test, Giemsa staining of microorganism, bacterial culture and 23S rRNA polymerase chain reaction. Past infection can be traced by using IgG anti-H. pylori antibody. Possibly past infection can reasonably assumed when the atrophic gastritis occurs following $H$. pylori associated chronic active gastritis. Because pepsinogen level decreases while gastric mucosa is atrophied, serum pepsinogen test is useful measure for atrophic gastritis and predictor for cancer recurrence. ${ }^{6}$ Serologic atrophy, pepsinogen I level $\leq 70 \mathrm{IU} / \mathrm{mL}$ or pepsinogen I/II ratio $\leq 3.0$, reliably indicate atrophic gastritis and possibly past $H$. pylori infection. ${ }^{7,8}$ Histological diagnosis using updated Sydney system also can tell the degree of atrophic gastritis and intestinal metaplasia.

Endoscopic diagnosis of atrophic gastritis which is usually made by using Kimura-Takemoto classification is on the center of debate regarding to its clinical value and reliability. While Japanese studies adopt endoscopic diagnosis of atrophic gastritis, ${ }^{4,5}$ Korean and Western studies do not. And there is a significant gap in the reported frequency of $H$. pylori-negative gastric cancer.

The last complicating issue comes from the cardia cancer of stomach. In general, $H$. pylori appear to be inversely associated with cardia cancer, and decreasing $H$. pylori prevalence seems to contribute to higher rate of cardia cancer in the Western. ${ }^{9}$ Cardia cancers are of two distinct types: type A resembles the gastric body cancer and a consequence of atrophic metaplastic gastritis by $H$. pylori infection; type B is like distal esophageal adenocarcinoma and a result of chronic gastroesophageal reflux. Therefore, if type B cardia cancer is included in gastric cancer analysis, the $H$. pylori-negative gastric cancer frequency can be overestimated. Fortunately in Korea, cancer arising from Barrett's esophagus is extremely rare, we might conveniently count all cardia cancer as gastric body cancer.

Because of the rarity of $H$. pylori-negative gastric cancer, most studies contained only a small number of cases and the analysis for clinical characteristics often failed to achieve statistical significance. However, trends can be read intuitionally. The clinical characteristics of $H$. pylori-negative gastric cancer can be summarized as following: ${ }^{10} \mathrm{H}$. pylori-negative gastric cancers (1) are diagnosed in younger age, often under 60 years old; (2) develop equally in both gender; (3) locate more frequently in proximal stomach and cardia; (4) present more advanced TNM stage, including advanced T stage; (5) have poor prognosis; (6) have higher proportion of diffuse type cancer; (7) have a higher proportion of undifferentiated type adenocarcinoma, especially signet ring cell carcinoma than $H$. pylori-positive gastric cancers. In this study, authors' failed to prove the difference in cancer characteristics. But authors' earlier study which included resectable and unresectable gastric cancer reported advanced TNM stage and T stage in H. pylori-negative gastric cancer. ${ }^{3}$ Are these differences in clinical characteristics form $H$. pylori infection itself? Fundamentally, all cancer results from genetic instability and gastric cancer does so. Comprehensive molecular characterization study categorized the gastric cancer as four subtypes; EBV positive, MSI, genomically stable (GS), and chromosomal instability (CIN). ${ }^{11}$ And this categorization has no relation to $H$. pylori infection. In this study, authors found that $H$. pylori-negative gastric cancer showed higher MSI-H and higher p53 overexpression, but the meaning is not understood. Genetic instability comes from several ways. A small proportion are familial and related to inherited genetic abnormalities that involve alternations in tumor suppressor genes, proto-oncogenes,

Table 1. Definition Tools for Helicobacter pylori Infection Positivity

\begin{tabular}{|c|c|c|c|}
\hline \multicolumn{2}{|c|}{ Timing of infection } & \multirow{2}{*}{$\begin{array}{c}\text { Generally accepted test } \\
\text { Giemsa or Warthin-Starry silver staining }\end{array}$} & \multirow[t]{2}{*}{ Test on debate } \\
\hline Current infection & Endoscopic & & \\
\hline & & Rapid urease test (CLOtest) & \\
\hline & & 23S rRNA PCR & \\
\hline & & Culture & \\
\hline & Noninvasive & Urea breath test & \\
\hline & & Stool Hp antigen & \\
\hline Past infection & Noninvasive & Serum anti-H. pylori antibody (IgG) & \\
\hline \multirow[t]{2}{*}{ Possibly past infection } & Endoscopic & $\begin{array}{l}\text { Histological examination of atrophy and } \\
\text { intestinal metaplasia }\end{array}$ & $\begin{array}{l}\text { Endoscopic diagnosis of } \\
\text { atrophic gastritis }\end{array}$ \\
\hline & Serologic & Pepsinogen I and II level & \\
\hline
\end{tabular}

CLO, campylobacter-like organism; PCR, polymerase chain reaction; Hp, Helicobacter pylori. 
gatekeeper genes, genes encoding enzymes, growth factors, membrane or nuclear receptors. Chronic infection, inflammation and irritation can also result in DNA damage and genetic instability and many cancers are inflammation-related. $H$. pylori itself and the oxidative stress from $H$. pylori infection both can result in gastric epithelial cell DNA damages, epigenetic changes and finally carcinogenesis. ${ }^{12}$ But unlike inherited genetic abnormalities, $H$. pylori induced genetic instability needs a long time and complex conditions to develop. Time and conditions including age, sex, possible cancer stem cell niche, and background mucosal integrity of $H$. pylori infected population can affect the clinical characteristics of $H$. pylori-positive gastric cancer. Better prognosis of $H$. pylori-positive gastric cancer than $H$. pylori-negative gastric cancer is considered due to its peritumoral inflammation, and the good prognosis of EBV associated lymphoepithelioma-like carcinoma (LELC) can be an example for this explanation.

Finally, the results of $H$. pylori-negative gastric cancer studies give a message that the most of gastric cancers, over 95\% at least, are initiated from $H$. pylori infection. In other word, we can expect effective avoidance of gastric cancer development after proper and timely $H$. pylori eradication. The relative rarity of $H$. pylori-negative gastric cancer may suggest that the population-screening EGD can be reasonably performed on only $H$. pylori infected individuals. Though $H$. pylori prevalence continues to decrease in future, the incidence of $H$. pylori-negative gastric cancer will be steady or even increase due to various reasons. We cannot take our eyes off the $H$. pylori-negative gastric cancer and, according to the clinical characteristics of $H$. pylori-negative gastric cancer, we may need to prepare a new strategy for cancer prevention, screening and treatment.

\section{CONFLICTS OF INTEREST}

No potential conflict of interest relevant to this article was reported.

\section{REFERENCES}

1. de Martel C, Ferlay J, Franceschi S, et al. Global burden of cancers attributable to infections in 2008: a review and synthetic analysis. Lancet Oncol 2012;13:607-615.

2. Kim HJ, Kim N, Yoon H, et al. Comparison between resectable Helicobacter pylori-negative and -positive gastric cancers. Gut Liver 2016;10:212-219.

3. Yoon H, Kim N, Lee HS, et al. Helicobacter pylori-negative gastric cancer in South Korea: incidence and clinicopathologic characteristics. Helicobacter 2011;16:382-388.

4. Matsuo T, Ito M, Takata S, Tanaka S, Yoshihara M, Chayama K. Low prevalence of Helicobacter pylori-negative gastric cancer among Japanese. Helicobacter 2011;16:415-459.

5. Ono S, Kato M, Suzuki M, et al. Frequency of Helicobacter pylorinegative gastric cancer and gastric mucosal atrophy in a Japanese endoscopic submucosal dissection series including histological, endoscopic and serological atrophy. Digestion 2012;86:59-65.

6. Park SY, Lim SO, Ki HS, et al. Low pepsinogen I level predicts multiple gastric epithelial neoplasias for endoscopic resection. Gut Liver 2014;8:277-281.

7. Kitahara F, Kobayashi K, Sato T, Kojima Y, Araki T, Fujino MA. Accuracy of screening for gastric cancer using serum pepsinogen concentrations. Gut 1999;44:693-697.

8. Miki K, Ichinose M, Ishikawa KB, et al. Clinical application of serum pepsinogen I and II levels for mass screening to detect gastric cancer. Jpn J Cancer Res 1993;84:1086-1090.

9. Kamangar F, Dawsey SM, Blaser MJ, et al. Opposing risks of gastric cardia and noncardia gastric adenocarcinomas associated with Helicobacter pylori seropositivity. J Natl Cancer Inst 2006;98: 1445-1452.

10. Yamamoto Y, Fujisaki J, Omae M, Hirasawa T, Igarashi M. Helicobacter pylori-negative gastric cancer: characteristics and endoscopic findings. Dig Endosc 2015;27:551-561.

11. Cancer Genome Atlas Research Network. Comprehensive molecular characterization of gastric adenocarcinoma. Nature 2014;513:202209

12. Lee K, Hwang H, Nam KT. Immune response and the tumor microenvironment: how they communicate to regulate gastric cancer. Gut Liver 2014;8:131-139. 